\title{
Descent theory of simple sheaves on $C_{1}$-fields
}

\author{
Ananyo Dan $\mathbb{D}$, Inder Kaur(D)
}

\begin{abstract}
Let $K$ be a $C_{1}$-field of any characteristic and $X$ a projective variety over $K$. In this article we prove that for a finite Galois extension $L$ of $K$, a simple sheaf with covering datum on $X \times_{K} L$ descends to a simple sheaf on $X$. As a consequence, we show that there is a $1-1$ correspondence between the set of geometrically stable sheaves on $X$ with fixed Hibert polynomial $P$ and the set of $K$-rational points of the corresponding moduli space.
\end{abstract}

\section{Introduction}

Let $f: Y \rightarrow X$ be a morphism of schemes and $\operatorname{pr}_{i}: Y \times_{X} Y \rightarrow Y$ the natural projection morphisms. For any quasi-coherent sheaf $\mathcal{F}$ on $Y$,

2000 AMS Subject Classification: Primary 12G05, 16K50, 14D20, 14J60, Secondary 14L24, 14D22.

Key Words and Phrases: Geometrically stable sheaves, Galois descent, Galois cohomology, Brauer group, $C_{1}$-fields.

The first author is currently supported by ERCEA Consolidator Grant 615655NMST and also by the Basque Government through the BERC 2014 - 2017 program and by Spanish Ministry of Economy and Competitiveness MINECO: BCAM Severo Ochoa excellence accreditation SEV-2013 - 0323. The second author is funded by a CAPES-PNPD fellowship. 
a covering datum of $\mathcal{F}$ is an isomorphism $\phi: \operatorname{pr}_{1}^{*} \mathcal{F} \rightarrow \operatorname{pr}_{2}^{*} \mathcal{F}$. If $f$ is faithfully flat and quasi-compact then the functor from the category of quasi-coherent sheaves on $X$ to the category of quasi-coherent sheaves on $Y$ with covering datum, induced by pull-back by $f$, is fully faithful (see $[1, \S 6.1$, Proposition 1]). Let us consider the simple case when $X$ is a projective variety over a field $K, L$ a finite Galois extension of $K$ and $Y:=X \times_{K} \operatorname{Spec}(L)$ is the base change of $X$ by the field extension $L$ of $K$. For a coherent sheaf $\mathcal{E}_{L}$ on $Y$, descent theory gives a criterion for when there exists a coherent sheaf $\mathcal{E}$ on $X$ such that $f^{*} \mathcal{E} \cong \mathcal{E}_{L}$. The descent datum associated to $\mathcal{E}_{L}$ consists of a covering datum satisfying a cocycle condition. In general, it is not possible to associate to $\mathcal{E}_{L}$ a descent datum, for example, if $\mathcal{E}_{L}$ is the structure sheaf of an $L$-point on $Y$ that does not come from a $K$-point of $X$, then there does not exist a descent datum associated to $\mathcal{E}_{L}$.

Recall that a field is called $C_{1}$ (pseudo-algebraically closed) if any degree $d$ polynomial in $n$ variables with coefficients in the field and $n>d$ has a non-trivial solution. These fields have been studied by Tsen [19], Lang [13], Chevalley [2], Greenberg [6], Grabber-Harris-Starr [5] and many others (see $[9, \S 2.1]$ for a short introduction to $C_{1}$ fields). In this article, we consider the case when $X$ is a projective variety over a $C_{1}$-field $K, L$ is a finite Galois extension of $K$ and $Y:=X \times_{K} \operatorname{Spec}(L)$. We call a simple (resp. geometrically stable) sheaf $\mathcal{E}_{L}$ on $Y, K$-simple (resp. $K$ geometrically stable) if one can associate a covering datum to $\mathcal{E}_{L}$ (see Definition 2.7).

We prove that:

Theorem 1.1. The natural functor from the category $\mathfrak{S}_{X}$ of simple sheaves on $X$ to the category $\mathfrak{S}_{\mathfrak{K}_{Y}}$ of $K$-simple sheaves on $Y$, defined by pull-back of sheaves via $f$, is an equivalence of categories.

Clearly, any coherent sheaf $\mathcal{E}_{L}$ with covering datum on $Y$ descends to $X$ if the covering datum satisfies the cocycle condition. However, we show that the cocycle condition is indeed satisfied if $\mathcal{E}_{L}$ is $K$-simple and 
$H^{2}\left(K, \mathbb{G}_{m}\right)$ vanishes (see proof of Proposition 2.8). Since $K$ is a $C_{1}$-field, the cohomology group $H^{2}\left(K, \mathbb{G}_{m}\right)$ is trivial (see [4, Proposition 6.2.3]). We then give an application of Theorem 1.1. Fix $P$ the Hilbert polynomial of a coherent sheaf on $X$ with rank coprime to degree. Denote by $M_{X}(P)$ the moduli scheme of geometrically stable sheaves on $X$ with Hilbert polynomial $P$. Recall, a point $x \in X$ is called a $K$-rational point if the corresponding residue field is contained in $K$. We show:

Theorem 1.2. There is a $1-1$ correspondence between the set of $K$ rational points of $M_{X}(P)$ and the set of geometrically stable sheaves on $X$ with Hilbert polynomial $P$.

We note that a similar result to Theorem 1.2 for stacks has been proven by Kraschen and Lieblich in [12, Proposition 1.1.5]. However their proof uses the theory of gerbes. In contrast our proof uses only basic algebraic geometry.

In $\S 4$ we give possible applications of the above theorems to the existence of rational points $\left(C_{1}\right.$-conjecture due to Lang, Manin and Kollár) and index of varieties.

Acknowledgements. A part of this work was done when she was visiting ICTP. She warmly thanks ICTP, the Simons Associateship and Prof. Carolina Araujo for making this possible.

\section{Galois action on simple sheaves}

We begin by recalling the basic definitions and results we need.

Definition 2.1. Let $f: S^{\prime} \rightarrow S$ be a morphism of schemes. Set $S^{\prime \prime}:=$ $S^{\prime} \times_{S} S^{\prime}, S^{\prime \prime \prime}:=S^{\prime} \times_{S} S^{\prime} \times_{S} S^{\prime}, \operatorname{pr}_{i}: S^{\prime \prime} \rightarrow S^{\prime}$ and $\operatorname{pr}_{i j}: S^{\prime \prime \prime} \rightarrow S^{\prime \prime}$ the natural projections onto the factors with indices $i$ and $j$, for $i<j$, $i, j \in\{1,2,3\}$. A descent datum on a quasi-coherent sheaf $\mathcal{F}$ on $S^{\prime}$ is a covering datum $\phi: \operatorname{pr}_{1}^{*} \mathcal{F} \stackrel{\sim}{\rightarrow} \operatorname{pr}_{2}^{*} \mathcal{F}$ on $\mathcal{F}$ which satisfies the cocycle condition $\operatorname{pr}_{13}^{*} \phi=\operatorname{pr}_{23}^{*} \phi \circ \operatorname{pr}_{12}^{*} \phi$ i.e., $\operatorname{pr}_{13}^{*} \phi$ coincides with the composition: $\mathrm{pr}_{13}^{*} \mathrm{pr}_{1}^{*} \mathcal{F} \cong \mathrm{pr}_{12}^{*} \mathrm{pr}_{1}^{*} \mathcal{F} \stackrel{\mathrm{pr}_{12}^{*} \phi}{\longrightarrow} \mathrm{pr}_{12}^{*} \mathrm{pr}_{2}^{*} \cong \mathrm{pr}_{23}^{*} \mathrm{pr}_{1}^{*} \mathcal{F} \stackrel{\mathrm{pr}_{23}^{*} \phi}{\longrightarrow} \mathrm{pr}_{23}^{*} \mathrm{pr}_{2}^{*} \mathcal{F} \cong \mathrm{pr}_{13}^{*} \mathrm{pr}_{2}^{*} \mathcal{F}$, 
where the three isomorphisms follow from $\mathrm{pr}_{1} \circ \mathrm{pr}_{13}=\mathrm{pr}_{1} \circ \mathrm{pr}_{12}, \mathrm{pr}_{1} \circ \mathrm{pr}_{23}=$ $\mathrm{pr}_{2} \circ \mathrm{pr}_{12}$ and $\mathrm{pr}_{2} \circ \mathrm{pr}_{23}=\mathrm{pr}_{2} \circ \mathrm{pr}_{13}$, respectively.

Proposition 2.2 ([1, $\S 6.1$, Proposition 1]). Notations as in Definition 2.1. Let $f: S^{\prime} \rightarrow S$ be a faithfully flat and quasi-compact morphism of schemes and $\mathcal{F}, \mathcal{G}$ be quasi-coherent $S$-modules and set $q:=f \circ \mathrm{pr}_{1}=f \circ \mathrm{pr}_{2}$. Then, identifying $q^{*} \mathcal{F}\left(\right.$ resp. $\left.q^{*} \mathcal{G}\right)$ canonically with $\operatorname{pr}_{i}^{*}\left(f^{*} \mathcal{F}\right)\left(\operatorname{resp} \operatorname{pr}_{i}^{*}\left(f^{*} \mathcal{G}\right)\right)$ for $i=1,2$, the sequence

$$
\operatorname{Hom}_{S}(\mathcal{F}, \mathcal{G}) \stackrel{f^{*}}{\longrightarrow} \operatorname{Hom}_{S^{\prime}}\left(f^{*} \mathcal{F}, f^{*} \mathcal{G}\right) \stackrel{\operatorname{pr}_{2}^{*}}{\longrightarrow} \operatorname{Hom}_{S^{\prime \prime}}\left(q^{*} \mathcal{F}, q^{*} \mathcal{G}\right)
$$

is exact. In other words, the functor $\mathcal{F} \mapsto f^{*} \mathcal{F}$ from quasi-coherent $S$ modules to quasi-coherent $S^{\prime}$-modules with covering datum is fully faithful.

We now recall the definition of semi-stable sheaves and simple sheaves.

Definition 2.3. Let $\mathcal{E}$ be a coherent sheaf with support of dimension $d$. The Hilbert polynomial $P(\mathcal{E})(t)$ of $\mathcal{E}$ can be expressed as (see [7, Lemma $1.2 .1])$

$$
P(\mathcal{E})(t):=\chi\left(\mathcal{E} \otimes \mathcal{O}_{X_{k}}(t)\right)=\sum_{i=0}^{d} \alpha_{i}(\mathcal{E}) \frac{t^{i}}{i !} \text { for } t>>0 .
$$

The reduced Hilbert polynomial is defined as $P_{\text {red }}(\mathcal{E})(t):=\frac{P(\mathcal{E})(t)}{\alpha_{d}(\mathcal{E})}$. The sheaf $\mathcal{E}$ is called Gieseker (semi)stable if for any proper subsheaf $\mathcal{F} \subset \mathcal{E}, P_{\text {red }}(\mathcal{F})(t)(\leq)<P_{\text {red }}(\mathcal{E})(t)$ for all $t$ large enough. In other words, $\mathcal{E}$ is (semi)stable if properly included subsheaves have (strictly) smaller reduced Hilbert polynomials.

Definition 2.4. A sheaf $\mathcal{E}$ defined on a projective variety defined over a field $k$ is called simple if $\operatorname{End}(E) \simeq k$.

Lemma 2.5 ([8, Corollary 1.2.8]). If $\mathcal{E}$ is a stable sheaf on a projective variety defined over an algebraically closed field, say $k$, then $\operatorname{End}(E) \simeq k$. 
Notation 2.6. Let $K$ be a $C_{1}$ field of any characteristic, $X$ a projective variety over $K$. Let $K \subset L$ be an algebraic field extension of $K$ (not necessarily finite). There exist natural morphisms

$$
\operatorname{pr}_{1, L}: L \rightarrow L \otimes_{K} L, \operatorname{pr}_{2, L}: L \rightarrow L \otimes_{K} L
$$

where

$$
\operatorname{pr}_{1, L}(a)=a \otimes 1 \text { and } \operatorname{pr}_{2, L}(a)=1 \otimes a .
$$

This induces morphisms

$$
\operatorname{pr}_{i, L}: X_{L \otimes_{K} L} \rightarrow X_{L} \text { for } i=1,2
$$

Denote by $G_{L}:=\operatorname{Gal}(L / K)$ the Galois group. For any $\sigma \in G_{L}$, we denote by

$$
\sigma: X_{L} \rightarrow X_{L}
$$

the induced natural morphism. Moreover, for $\sigma, \tau \in G_{L}$, we have $\sigma \tau$ : $L \stackrel{\tau}{\rightarrow} L \stackrel{\sigma}{\rightarrow} L$. As taking spectrum is contravariant, this induces $(\sigma \tau)$ : $X_{L} \stackrel{\sigma}{\rightarrow} X_{L} \stackrel{\tau}{\rightarrow} X_{L}$. Thus, for any coherent sheaf $\mathcal{E}$ on $X_{L}$, the pull-back $(\sigma \tau)^{*} \mathcal{E}=\left(\sigma^{*} \circ \tau^{*}\right) \mathcal{E}$. In the case $L=\bar{K}$, the algebraic closure, denote by $\operatorname{pr}_{i}:=\operatorname{pr}_{i, \bar{K}}$ and $G:=G_{\bar{K}}$.

Definition 2.7. Recall, a sheaf $\mathcal{E}$ on $X_{L}$ is called geometrically stable if for any field extension $L^{\prime}$ of $L$, the sheaf $\mathcal{E} \otimes_{L} L^{\prime}$ is stable over $X_{L} \times{ }_{L} \operatorname{Spec}\left(L^{\prime}\right)$. We call a simple (resp. geometrically stable) sheaf $\mathcal{E}$ on $X_{L}, K$-simple (resp. K-geometrically stable) if there exists an isomorphism $\psi: \operatorname{pr}_{1, L}^{*} \mathcal{E} \rightarrow$ $\operatorname{pr}_{2, L}^{*} \mathcal{E}$. In other words, a $K$-simple (resp. $K$-geometrically stable) sheaf is a simple (resp. geometrically stable) sheaf on $X_{L}$ such that one can associate to it a covering datum.

We now study the action of the Galois group $G_{L}$ on a simple sheaf $\mathcal{E}$ on $X_{L}$. We observe that in the case $L$ is a finite Galois extension of $K, \mathcal{E}$ descends to $X$ if and only if it is $K$-simple (Theorem 2.9). 
Proposition 2.8. Let $\mathcal{E}$ be a $K$-simple sheaf on $X_{L}$. Then, there exists a collection $\left(\lambda_{\sigma}\right)_{\sigma \in G_{L}}$ of isomorphisms $\lambda_{\sigma}: \mathcal{E} \rightarrow \sigma^{*} \mathcal{E}$ satisfying the cocycle condition:

$$
\left(\sigma^{*} \lambda_{\tau}\right) \circ \lambda_{\sigma}=\lambda_{\sigma \tau} \text { for any pair } \sigma, \tau \in G_{L}
$$

Proof. Fix $\sigma \in G_{L}$. Consider the homomorphism $L \otimes_{K} L \rightarrow L$ defined by $a \otimes b$ maps to $a \sigma(b)$. This induces a natural morphism

$$
p_{\sigma}: X_{L} \rightarrow X_{L \otimes_{K} L}
$$

Observe that the morphism $p_{\sigma}$ has the property that its composition with $\operatorname{pr}_{1, L}$

$$
X_{L} \stackrel{p_{\sigma}}{\longrightarrow} X_{L \otimes_{K} L} \stackrel{\mathrm{pr}_{1, L}}{\longrightarrow} X_{L}
$$

is simply the identity map and with $\operatorname{pr}_{2, L}$,

$$
X_{L} \stackrel{p_{\sigma}}{\longrightarrow} X_{L \otimes_{K} L} \stackrel{\mathrm{pr}_{2, L}}{\longrightarrow} X_{L}
$$

is the morphism $\sigma: X_{L} \rightarrow X_{L}$. Then, $\operatorname{pr}_{1, L}^{*} \mathcal{E} \cong \operatorname{pr}_{2, L}^{*} \mathcal{E}$ implies that

$$
\mathcal{E} \cong p_{\sigma}^{*} \operatorname{pr}_{1, L}^{*} \mathcal{E} \cong p_{\sigma}^{*} \operatorname{pr}_{2, L}^{*} \mathcal{E} \cong \sigma^{*} \mathcal{E}
$$

Therefore, for any $\sigma \in G_{L}$, there exists an isomorphism $\lambda_{\sigma}^{\prime}: \mathcal{E} \rightarrow \sigma^{*} \mathcal{E}$. Let $\tau \in G_{L}$. Since $\mathcal{E}$ is simple,

$$
\operatorname{End}\left(\sigma^{*} \tau^{*} \mathcal{E}\right) \cong \sigma^{*} \tau^{*} \operatorname{End}(\mathcal{E}) \cong L
$$

Hence, there exists $a_{\sigma, \tau} \in L^{\times}=\operatorname{Aut}\left(\sigma^{*} \tau^{*} \mathcal{E}\right)$ such that the following diagram is commutative:

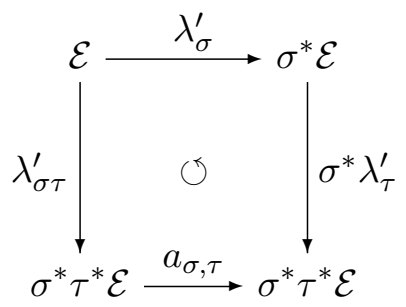


This directly implies the following equalities: Given $g_{1}, g_{2}, g_{3} \in G_{L}$, we have

$$
\begin{aligned}
a_{g_{1},\left(g_{2} g_{3}\right)} \circ \lambda_{g_{1}\left(g_{2} g_{3}\right)}^{\prime} & =\left(g_{1}^{*} \lambda_{g_{2} g_{3}}^{\prime}\right) \circ \lambda_{g_{1}}^{\prime} \\
g_{1}^{*} a_{g_{2} g_{3}} \circ g_{1}^{*} \lambda_{g_{2} g_{3}}^{\prime} & =\left(g_{1}^{*} g_{2}^{*} \lambda_{g_{3}}^{\prime}\right) \circ g_{1}^{*} \lambda_{g_{2}}^{\prime} \\
a_{g_{1}, g_{2}} \circ \lambda_{g_{1} g_{2}}^{\prime} & =\left(g_{1}^{*} \lambda_{g_{2}}^{\prime}\right) \circ \lambda_{g_{1}}^{\prime} \\
\left(\left(g_{1} g_{2}\right)^{*} \lambda_{g_{3}}^{\prime}\right) \circ \lambda_{g_{1} g_{2}}^{\prime} & =a_{\left(g_{1} g_{2}\right), g_{3}} \circ \lambda_{\left(g_{1} g_{2}\right) g_{3}}^{\prime}
\end{aligned}
$$

Applying $g_{1}^{*} a_{g_{2}, g_{3}} \circ-$ to both sides of $(2.1)$, we get,

$$
\begin{aligned}
g_{1}^{*} a_{g_{2}, g_{3}} \circ a_{g_{1},\left(g_{2} g_{3}\right)} \circ \lambda_{g_{1}\left(g_{2} g_{3}\right)}^{\prime} & =g_{1}^{*} a_{g_{2}, g_{3}} \circ\left(g_{1}^{*} \lambda_{g_{2} g_{3}}^{\prime}\right) \circ \lambda_{g_{1}}^{\prime} \\
& =\left(g_{1}^{*} g_{2}^{*} \lambda_{g_{3}}^{\prime}\right) \circ g_{1}^{*} \lambda_{g_{2}}^{\prime} \circ \lambda_{g_{1}}^{\prime} \quad \text { by }(2.2) \\
& =\left(g_{1}^{*} g_{2}^{*} \lambda_{g_{3}}^{\prime}\right) \circ a_{g_{1}, g_{2}} \circ \lambda_{g_{1} g_{2}}^{\prime} \quad \text { by }(2.3) \\
& =a_{g_{1}, g_{2}} \circ a_{\left(g_{1} g_{2}\right), g_{3}} \circ \lambda_{g_{1} g_{2} g_{3}}^{\prime} \quad \text { by }(2.4)
\end{aligned}
$$

where the last equality follows from the fact that multiplication by a scalar $a_{g_{1}, g_{2}}$ commutes with $\left(g_{1}^{*} g_{2}^{*} \lambda_{g_{3}}^{\prime}\right)$. Since $\lambda_{g_{1} g_{2} g_{3}}^{\prime}$ is an isomorphism, we have the 2-cocycle condition:

$$
g_{1}^{*} a_{g_{2}, g_{3}} \circ a_{g_{1},\left(g_{2} g_{3}\right)}=a_{g_{1}, g_{2}} \circ a_{\left(g_{1} g_{2}\right), g_{3}} .
$$

Since $K$ is a $C_{1}$ field, $H^{2}\left(K, \mathbb{G}_{m}\right)=0$ (see [16, p. 161, Proposition 10]). This means that for any sequence $\left(a_{\sigma, \tau}\right)_{\sigma, \tau \in G_{L}}$ satisfying the 2-cocycle condition there exists a continuous morphism $\phi: G_{L} \rightarrow L^{\times}$such that

$$
a_{\sigma, \tau}=\sigma \phi(\tau) \phi(\sigma \tau)^{-1} \phi(\sigma)
$$

Consider now the isomorphism given by

$$
\lambda_{\sigma}:=\phi(\sigma)^{-1} \lambda_{\sigma}^{\prime}: \mathcal{E} \stackrel{\lambda_{\sigma}^{\prime}}{\longrightarrow} \sigma^{*} \mathcal{E} \stackrel{\phi(\sigma)^{-1}}{\longrightarrow} \sigma^{*} \mathcal{E}, \text { for all } \sigma \in G_{L} .
$$

Since $\phi(\sigma)$ is scalar, it commutes with $\lambda_{\sigma \tau}^{\prime}$ i.e., we have the following 
commutative diagram:

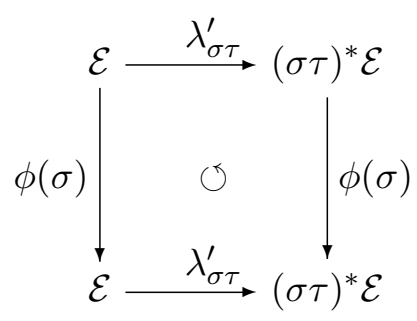

Using (2.3) and substituting for $a_{\sigma, \tau}$, we conclude that $\sigma^{*} \lambda_{\tau}^{\prime} \circ \lambda_{\sigma}^{\prime}$ equals

$$
a_{\sigma, \tau} \circ \lambda_{\sigma \tau}^{\prime}=\sigma \phi(\tau) \phi(\sigma \tau)^{-1} \phi(\sigma) \lambda_{\sigma \tau}^{\prime}=\sigma \phi(\tau) \phi(\sigma \tau)^{-1} \lambda_{\sigma \tau}^{\prime} \phi(\sigma) .
$$

Therefore,

$$
\left(\sigma^{*} \lambda_{\tau}\right)=(\sigma \phi(\tau))^{-1} \sigma^{*} \lambda_{\tau}^{\prime}=\left(\phi(\sigma \tau)^{-1} \lambda_{\sigma \tau}^{\prime}\right) \circ\left(\phi(\sigma)^{-1} \lambda_{\sigma}^{\prime}\right)^{-1}=\lambda_{\sigma \tau} \circ \lambda_{\sigma}^{-1} .
$$

Hence, $\left(\sigma^{*} \lambda_{\tau}\right) \circ \lambda_{\sigma}=\lambda_{\sigma \tau}$. This proves the proposition.

Theorem 2.9. Let $L$ be a finite Galois extension of $K$ and $f: X_{L} \rightarrow X$ the natural morphism. The natural functor from the category $\mathfrak{S}_{X}$ of simple sheaves on $X$ to the category $\mathfrak{S} \mathfrak{K}_{X_{L}}$ of $K$-simple sheaves on $X_{L}$, defined by pull-back of sheaves via $f$, is an equivalence of categories.

Proof. It suffices to show that any simple sheaf $\mathcal{E}_{L}$ on $X_{L}$ is $K$-simple if and only if there exists a simple sheaf $\mathcal{E}$ on $X$ such that $f^{*} \mathcal{E} \cong \mathcal{E}_{L}$. By Proposition 2.2, for any simple sheaf $\mathcal{E}$ on $X$, we have $f^{*} \mathcal{E}$ is $K$-simple on $X_{L}$. We now prove the converse. Let $\mathcal{E}_{L}$ be $K$-simple on $X_{L}$. Proposition 2.8 implies that there exists a collection $\left(\lambda_{\sigma}\right)_{\sigma \in G_{L}}$ of isomorphisms $\lambda_{\sigma}$ : $\mathcal{E}_{L} \rightarrow \sigma^{*} \mathcal{E}_{L}$ such that $\left(\sigma^{*} \lambda_{\tau}\right) \circ \lambda_{\sigma}=\lambda_{\sigma \tau}$ for any pair $\sigma, \tau \in G_{L}$. By Galois descent, this implies that there exists a coherent sheaf $\mathcal{E}$ on $X$ such that $\mathcal{E}_{L} \cong f^{*} \mathcal{E}$. Since $f$ is flat,

$$
f^{*}(\operatorname{End}(\mathcal{E})) \cong \operatorname{End}\left(\mathcal{E}_{L}\right) \cong L
$$

As $\operatorname{End}(\mathcal{E})$ is a $K$-vector space, this directly implies that $\operatorname{End}(\mathcal{E}) \cong K$ i.e., $\mathcal{E}$ is simple. This proves the theorem. 


\section{Moduli of $K$-geometrically stable sheaves}

Keep Notations 2.6. In this section we use Theorem 2.9 to prove that the set of $K$-rational points of the moduli space of geometrically stable sheaves on $X$ is in 1-1 correspondence with the set of geometrically stable sheaves on $X$.

Recall, the definition of the moduli functor of semi-stable sheaves over a projective variety $X$.

Definition 3.1. Fix $P$ the Hilbert polynomial of a coherent sheaf on $X$ with rank coprime to degree. Denote by $\mathcal{M}_{X}(P)$ the moduli functor:

$$
\mathcal{M}_{X}(P):\{\operatorname{Sch} / K\}^{\circ} \rightarrow \text { Sets }
$$

such that for a $K$-scheme $T$,

$\mathcal{M}_{X}(P)(T):=\left\{\begin{array}{l}\text { isomorphism classes of pure sheaves } \mathcal{F} \text { on } X \times T \text { flat } \\ \text { over } T \text { and for every geometric point } t \in T,\left.\mathcal{F}\right|_{X_{t}} \\ \text { is a stable sheaf with Hilbert polynomial } P \text { on } X_{t}\end{array}\right\} / \sim$

where $\mathcal{F} \sim \mathcal{G}$ if there exists an invertible sheaf $\mathcal{L}$ on $T$ such that $\mathcal{F} \cong$ $\mathcal{G} \otimes p_{T}^{*} \mathcal{L}$, where $p_{T}: X_{T} \rightarrow T$ is the natural projection map.

Theorem 3.2 ([14, Theorem 4.1]). Let $R$ be a universally Japanese ring and $f: X \rightarrow S$ a projective morphism of $R$-schemes of finite type with geometrically connected fibres, and let $\mathcal{O}_{X}(1)$ be an $f$-ample line bundle. Then, for a fixed polynomial $P$, there exists a projective $S$-scheme $M_{X / S}(P)$ of finite type over $S$ which uniformly corepresents the functor

$$
\mathcal{M}_{X / S}(P):\{\operatorname{Sch} / S\}^{\circ} \rightarrow \text { Sets }
$$

such that for a $S$-scheme $T$,

$\mathcal{M}_{X / S}(P)(T):=\left\{\begin{array}{c}S \text { equivalence classes of pure sheaves } \mathcal{F} \text { on } X \times_{S} T \text { flat } \\ \text { over } T \text { such that for every geometric point } t \in T,\left.\mathcal{F}\right|_{X_{t}} \\ \text { is a semi-stable sheaf with Hilbert polynomial } P \text { on } X_{t}\end{array}\right\} / \sim$

where $\mathcal{F} \sim \mathcal{G}$ if there exists an invertible sheaf $\mathcal{L}$ on $T$ such that $\mathcal{F} \cong$ $\mathcal{G} \otimes p_{T}^{*} \mathcal{L}$, where $p_{T}: X \times_{S} T \rightarrow T$ is the natural projection map. 
Moreover, there is an open subscheme $M_{X / S}^{s}(P)$ of $M_{X / S}(P)$ which universally corepresents the subfunctor of families of geometrically stable sheaves.

Remark 3.3. Note that a $C_{1}$-field $K$ is a universally Japanese ring. If the rank and degree of coherent sheaves with Hilbert polynomial $P$, are coprime, then stability and semi-stability coincide (see [8, Lemmas 1.2.13 and 1.2.14]). Then by Theorem 3.2, there exists a projective $K$-scheme of finite type $M_{X}(P)$, universally corepresenting the functor $\mathcal{M}_{X}(P)$.

We now review briefly the construction of the moduli scheme $M_{X}(P)$. By [14, Theorem 4.2], there exists an integer $e$ such that any semi-stable sheaf on $X$ with Hilbert polynomial $P$ is $e$-regular (in the sense of Castelnuovo-Mumford regularity). Fix such an integer $e$. Denote by $\mathcal{H}:=\mathcal{O}_{X}(-e)^{\oplus P(e)}$ and by $\operatorname{Quot}_{\mathcal{H} / X / P}$ the Quot scheme parametrizing all quotients of the form $\mathcal{H} \rightarrow \mathcal{Q}_{0}$, where $\mathcal{Q}_{0}$ has Hilbert polynomial $P$ (see $[15, \S 4.4]$ for more details).

Let $\mathcal{R}$ be the subset of $\operatorname{Quot}_{\mathcal{H} / X / P}$ consisting of all points which parametrize quotients of the form $\mathcal{H} \rightarrow \mathcal{Q}_{0}$ such that $\mathcal{Q}_{0}$ is semi-stable and $H^{0}\left(\mathcal{Q}_{0}(e)\right)$ is (non-canonically) isomorphic to $k^{\oplus P(e)}$. Now, semistability is an open condition (see [8, Proposition 2.3.1]). Therefore, $\mathcal{R}$ is an open subscheme in $\operatorname{Quot}_{\mathcal{H} / X / P}$. The group $\operatorname{GL}(P(e))=\operatorname{Aut}(\mathcal{H})$ acts on $\operatorname{Quot}_{\mathcal{H} / X / P}$ from the right by the composition $[\rho] \circ g=[\rho \circ g]$, where $[\rho: \mathcal{H} \rightarrow \mathcal{F}] \in \operatorname{Quot}_{\mathcal{H} / X / P}$ and $g \in \mathrm{GL}(P(e))$. By [14, Theorem 4.3], $\mathcal{R}$ is the set of semi-stable points of $\operatorname{Quot}_{\mathcal{H} / X / P}$ under this group action. The moduli scheme $M_{X}(P)$ of semi-stable sheaves on $X$ with Hilbert polynomial $P$ is the geometric quotient of $\mathcal{R}$ under this action (see [14, pp. 582, after Theorem 4.3]). Denote by

$$
\pi: \mathcal{R} \rightarrow M_{X}(P)
$$

the corresponding quotient morphism. The quotient exists due to Seshadri's result [17, Theorem 4]. 
Theorem 3.4. There is a $1-1$ correspondence between the set of $K$ rational points of $M_{X}(P)$ and the set of isomorphism classes of geometrically stable sheaves on $X$ with Hilbert polynomial $P$.

Proof. Let $x: \operatorname{Spec}(K) \rightarrow M_{X}(P)$ be a $K$-rational point of $M_{X}(P)$. Denote by $\mathcal{R}_{K}$ the base change of the morphism $\pi$ in (3.1) by the morphism $x$. Let $y \in \mathcal{R}_{K}$ be a closed point. Then $y$ is a $L$-rational point on $\mathcal{R}_{K}$ for some finite extension $L$ of $K$. Without loss of generality assume that $L$ is a finite Galois extension of $K$. Since the Quot functor is representable, the point $y$ corresponds to a quotient $\phi_{y}: \mathcal{H}_{L} \rightarrow \mathcal{E}_{L}$ on $X_{L}:=X \times_{K}$ $\operatorname{Spec}(L)$, where $\mathcal{H}_{L} \cong \mathcal{H} \otimes_{K} L$ and $\mathcal{E}_{L}$ is geometrically stable with Hilbert polynomial $P$ on $X_{L}$. For any $\sigma \in \operatorname{Gal}(L / K)$ and the induced morphism $f_{\sigma}: X_{L} \stackrel{\operatorname{id}_{X} \times \sigma}{\longrightarrow} X_{L}$, denote by $f_{\sigma}^{*} \phi_{y}$ the quotient morphism,

$$
\mathcal{H}_{L} \cong f_{\sigma}^{*} \mathcal{H}_{L} \rightarrow f_{\sigma}^{*} \mathcal{E}_{L}
$$

By the universal property of Quot scheme, $f_{\sigma}^{*} \phi_{y}$ corresponds to the composed morphism:

$$
\operatorname{Spec}(L) \stackrel{\sigma}{\rightarrow} \operatorname{Spec}(L) \stackrel{y}{\rightarrow} \mathcal{R}_{K}
$$

Since $\mathcal{R}_{K}$ is a fiber to the morphism $\pi$, this implies $f_{\sigma}^{*} \mathcal{E}_{L} \cong \mathcal{E}_{L}$. As a result we obtain a set $\left(\lambda_{\sigma}\right)_{\sigma \in \operatorname{Gal}(L / K)}$ of isomorphisms $\lambda_{\sigma}: \mathcal{E}_{L} \rightarrow \sigma^{*} \mathcal{E}_{L}$.

Consider now the morphism

$$
\Phi: L \otimes_{K} L \rightarrow \coprod_{\sigma \in \operatorname{Gal}(L / K)} L, \text { defined by } a \otimes b \mapsto(a \sigma(b))_{\sigma \in \operatorname{Gal}(L / K)} \text {. }
$$

It is easy to check that $\Phi$ is an isomorphism. Consider now the induced morphisms,

$$
\Phi_{i}: \coprod_{\sigma \in \operatorname{Gal}(L / K)} X_{L} \stackrel{\Phi}{\rightarrow} X_{L \otimes_{K} L} \stackrel{\mathrm{pr}_{i, L}}{\rightarrow} X_{L} \text { for } i=1,2 .
$$

Observe that

$$
\Phi_{1}=\coprod \text { id and } \Phi_{2}=\coprod_{\sigma \in \operatorname{Gal}(L / K)} f_{\sigma}
$$


where $f_{\sigma}: X_{L} \stackrel{\operatorname{id}_{X} \times \sigma}{\longrightarrow} X_{L}$. The set of isomorphims $\left(\lambda_{\sigma}\right)_{\sigma \in \operatorname{Gal}(L / K)}$ then induce an isomorphism $\psi: \Phi_{1}^{*} \mathcal{E}_{L} \stackrel{\sim}{\rightarrow} \Phi_{2}^{*} \mathcal{E}_{L}$. Since $\Phi$ is an isomorphism, $\psi$ induces an isomorphism $\operatorname{pr}_{1, L}^{*} \mathcal{E}_{L} \stackrel{\sim}{\rightarrow} \operatorname{pr}_{2, L}^{*} \mathcal{E}_{L}$ i.e., $\mathcal{E}_{L}$ is $K$-geometrically stable.

Since $\mathcal{E}_{L}$ is geometrically stable, $\mathcal{E}_{\bar{K}}:=\mathcal{E}_{L} \otimes_{L} \bar{K}$ is stable. By Lemma $2.5, \mathcal{E}_{\bar{K}}$ is simple. Since $\operatorname{End}\left(\mathcal{E}_{L}\right)$ is an $L$-vector space and

$$
\operatorname{End}\left(\mathcal{E}_{L}\right) \otimes_{L} \bar{K} \cong \operatorname{End}\left(\mathcal{E}_{\bar{K}}\right) \cong \bar{K}
$$

we conclude that $\operatorname{End}\left(\mathcal{E}_{L}\right) \cong L$ i.e., $\mathcal{E}_{L}$ is simple. In particular, $\mathcal{E}_{L}$ is $K$ simple. Using Theorem 2.9, there exists a simple sheaf $\mathcal{E}$ on $X$ such that $\mathcal{E} \otimes_{K} L \cong \mathcal{E}_{L}$. By [8, Theorem 1.3.7], it follows that $\mathcal{E}$ is geometrically stable.

Conversely, by Theorem 3.2, any geometrically stable sheaf on $X$ with Hilbert polynomial $P$ corresponds to a $K$-rational point on $M_{X}(P)$. It is easy to check that the geometrically stable sheaf $\mathcal{E}$ corresponds to the $K$ rational point $x$ of $M_{X}(P)$. This gives us a $1-1$ correspondence between the $K$-rational points of $M_{X}(P)$ and the set of geometrically stable sheaves on $X$ with Hilbert polynomial $P$. This proves the theorem.

\section{Applications of descent theory}

In this section, we mention the application of descent theory studied before. Recall, the definition of a $C_{1}$ field given in the introduction.

Example 4.1. We state without proof some examples of $C_{1}$ fields:

1. An algebraically closed field is trivially $C_{1}$.

2. Finite fields are $C_{1}$ (see [2]).

3. The function field of an irreducible curve defined over an algebraically closed field is $C_{1}$ (see [19]).

4. Let $R$ be a Henselian discrete valuation ring of characteristic 0 with residue field denoted $k$, of characteristic $p$ and fraction field denoted $K$. If $k$ is algebraically closed, then $K$ is $C_{1}$ (see [13, Theorem 14]). 
Definition 4.2. A variety $Y$ over an algebraically closed field $\bar{K}$ is separably rationally connected if there exists a morphism $f: \mathbf{P}^{1} \rightarrow Y$ such that $f^{*}\left(T_{Y}\right)$ is ample.

Remark 4.3. Note that over an algebraically closed field $\bar{K}$ of characteristic 0 , rationally connected is equivalent to separably rationally connected (see [11, Proposition $I V .3 .3 .1]$ ).

The $C_{1}$ conjecture (Lang-Manin-Kollár): A smooth, proper, separably rationally connected variety over a $C_{1}$ field always has a rational point.

The conjecture has already been proven for various $C_{1}$ fields (see $[9$, Chapter 2] for a complete list).

Remark 4.4. The conjecture remains open in the case when the $C_{1}$ field is the fraction field of a maximal unramified discrete valuation ring with algebraically closed residue field of mixed characteristic.

Recently, the conjecture was shown to hold trivially for certain rationally connected varieties over such fields (see [10]). Let $M_{X_{K}, \mathcal{L}_{K}}^{s}(r, d)$ be the moduli space of geometrically stable locally free sheaves of rank $r$ and determinant $\mathcal{L}_{K}$. Denote by $M_{X_{\bar{K}}, \mathcal{L}_{\bar{K}}}^{s}(r, d)$ the moduli space of geometrically stable locally free sheaves of rank $r$ and determinant $\mathcal{L}_{\bar{K}}:=\mathcal{L}_{K} \otimes_{K} \bar{K}$ over the curve $X_{\bar{K}}:=X_{K} \times_{K} \operatorname{Spec}(\bar{K})$. By [18], $M_{X_{\bar{K}}, \mathcal{L}_{\bar{K}}}^{s}(r, d)$ is a unirational variety and therefore rationally connected. Since the moduli space $M_{X_{\bar{K}}, \mathcal{L}_{\bar{K}}}^{s}(r, d)$ is the base change $M_{X_{K}, \mathcal{L}_{K}}^{s}(r, d) \times_{K} \operatorname{Spec}(\bar{K})$, this implies $M_{X_{K}, \mathcal{L}_{K}}^{s}(r, d)$ is rationally connected. Suppose that $K$ is the fraction field of a Henselian discrete valuation ring with algebraically closed residue field. Then, $M_{X_{K}, \mathcal{L}_{K}}^{s}(r, d)$ has a $K$-rational point. This is shown using descent theory of stable sheaves on smooth, projective curves ([10, Theorem 1.2]). It is natural to ask how the descent theory of simple sheaves over $C_{1}$ fields can be used to prove the existence of rational points, in the higher dimension case. We cite some recent results in this direction.

Definition 4.5. Recall, the index of $X$, denoted ind $(X)$, is the gcd of the set of degrees of zero dimensional cycles on $X$. 
Remark 4.6. Clearly, the notion of index generalizes the idea of having a rational point. Applying Theorem 2.9 to the case of invertible sheaves on varieties, one can obtain a sufficient criterion for any projective variety defined over a $C_{1}$ field to have index 1 .

Definition 4.7. Denote by $G$ the absolute Galois group $\operatorname{Gal}(\bar{K} / K)$. An invertible sheaf $\mathcal{L}_{\bar{K}}$ on $X_{\bar{K}}:=X \times_{K} \operatorname{Spec}(\bar{K})$ is called $G$-invariant if for any $\sigma \in G$ and the corresponding morphism $\sigma: X_{\bar{K}} \rightarrow X_{\bar{K}}$, we have $\sigma^{*} \mathcal{L}_{\bar{K}} \cong \mathcal{L}_{\bar{K}}$. Denote by $\Lambda \subset \operatorname{Pic}\left(X_{\bar{K}}\right)$ the subgroup of $\operatorname{Pic}\left(X_{\bar{K}}\right)$ consisting of all $G$-invariant invertible sheaves on $X_{\bar{K}}$. Denote by $e:=$ $\operatorname{gcd}\left\{\chi\left(\mathcal{L}_{\bar{K}}\right) \mid \mathcal{L}_{\bar{K}} \in \Lambda\right\}$. We call $e$ the linear index of $X$, denoted lin $-\operatorname{ind}(X)$.

Theorem 4.8. Suppose that $H^{1}\left(\mathcal{O}_{X}\right)=0, \operatorname{Pic}\left(X_{\bar{K}}\right)$ is of rank $r$, generated by $\mathcal{L}_{1}, \ldots, \mathcal{L}_{r-1}$ and $\mathcal{L}_{r}:=H_{\bar{K}}=H \otimes_{K} \bar{K}$ satisfying the following conditions:

1. the ideal $\left(\operatorname{deg}\left(\mathcal{L}_{1}\right), \operatorname{deg}\left(\mathcal{L}_{2}\right), \ldots, \operatorname{deg}\left(\mathcal{L}_{r}\right)\right)$ in $\mathbb{Z}$ generated by $\operatorname{deg}\left(\mathcal{L}_{i}\right)$ for $i=1, \ldots, r$ coincides with the ideal (1),

2. for any $r \times r$-matrix $A=\left(a_{i, j}\right)$ with integral entries $a_{i, j}, a_{r, k}=0$ for all $k<r, a_{r, r}=1, A \neq \mathrm{Id}$ and $A^{t}=\mathrm{Id}$ for some $t>0$, we have $\sum_{j} a_{i j} \operatorname{deg}\left(\mathcal{L}_{j}\right) \neq \operatorname{deg}\left(\mathcal{L}_{i}\right)$ for some $i>0$.

Then, each $\mathcal{L}_{i}$ is $G$-invariant,

$$
\operatorname{lin}-\operatorname{ind}(X)=\operatorname{gcd}\left\{\chi\left(\mathcal{L}_{i}(n)\right) \mid i=1, \ldots, r \text { and } n \in \mathbb{Z}\right\}=1
$$

and

$$
\operatorname{ind}(X)=1 \text { if } \operatorname{char}(k)=0
$$

and

prime-to-p part of $\operatorname{ind}(X)$ equals 1 if $\operatorname{char}(k)=p>0$.

By prime-to-p part of $N$ we mean the largest divisor of $N$ which is prime to $p$.

Proof. See [3] for the proof. 
As a consequence of Theorem 4.8, we obtain numerous examples of smooth, projective varieties on $C_{1}$-fields with index 1 .

Example 4.9. Let $X$ be a smooth, projective variety with $\operatorname{deg}\left(H_{\bar{K}}\right)>2$, $H^{1}\left(\mathcal{O}_{X}\right)=0, \operatorname{Pic}\left(X_{\bar{K}}\right)$ is of rank 2 and there exists an invertible sheaf $\mathcal{L}_{0}$ of degree coprime to $\operatorname{deg}\left(H_{\bar{K}}\right)$ (for example, a smooth surface $X$ in $\mathbb{P}_{K}^{3}$ of degree at least 3 with $\operatorname{rk}\left(\operatorname{Pic}\left(X_{\bar{K}}\right)\right)=2$ and $X_{\bar{K}}$ contains a curve of degree coprime to $\operatorname{deg}(X))$. Theorem 4.8 implies that every invertible sheaf on $X_{\bar{K}}$ is $G$-invariant and $\operatorname{ind}(X)=\operatorname{lin}-\operatorname{ind}(X)=1$.

\section{References}

[1] S. Bosch, W. Lütkebohmert, and M. Raynaud. Néron models, volume 21. Springer Science \& Business Media, 2012.

[2] C. Chevalley. Démonstration d'une hypothèse de M. Artin. Abh. Math. Semin. Univ. Hamb., 11:73-75, 1935.

[3] A. Dan and I. Kaur. Examples of varieties with index one on $C_{1}$-fields. Journal of Number Theory, 203:242-248, 2019.

[4] P. Gille and T. Szamuely. Central simple algebras and Galois cohomology, volume 165. Cambridge University Press, 2017.

[5] T. Graber, J. Harris, and J. Starr. Families of rationally connected varieties. Journal of the American Mathematical Society, pages 5767, 2003.

[6] M. J. Greenberg. Lectures on forms in many variables, volume 31. W. A. Benjamin New York-Amsterdam, 1969.

[7] B. Hassett and K. Lai. Cremona transformations and derived equivalences of K3 surfaces. Compositio Mathematica, 154(7):1508-1533, 2018.

[8] D. Huybrechts and M. Lehn. The geometry of moduli spaces of sheaves. Springer, 2010. 
[9] I. Kaur. The $C_{1}$ conjecture for the moduli space of stable vector bundles with fixed determinant on a smooth projective curve. Ph. d. thesis, Freie University Berlin, 2016.

[10] I. Kaur. A pathological case of the $C_{1}$ conjecture in mixed characteristic. Mathematical Proceedings of the Cambridge Philosophical Society, 167(1):61-64, 2019.

[11] J. Kollár. Rational curves on algebraic varieties, volume 32. Springer Science \& Business Media, 2013.

[12] D. Krashen and M. Lieblich. Index reduction for brauer classes via stable sheaves. International Mathematics Research Notices, 2008.

[13] S. Lang. On quasi algebraic closure. Annals of Mathematics, pages 373-390, 1952.

[14] A. Langer. Moduli spaces of sheaves in mixed characteristic. Duke Mathematical Journal, 124(3):571-586, 2004.

[15] E. Sernesi. Deformations of Algebraic Schemes. Grundlehren der Mathematischen Wissenschaften-334. Springer-Verlag, 2006.

[16] J. P. Serre. Local fields, volume 67. Springer Science \& Business Media, 2013.

[17] C. S. Seshadri. Geometric reductivity over arbitrary base. Advances in Mathematics, 26(3):225-274, 1977.

[18] C. S. Seshadri. Fibrés vectoriels sur les courbes algébriques: conférences à l'ENS, Juin 1980. Number 95-96. Société Mathématique de France, 1982.

[19] C. Tsen. Divisionsalgebren über Funktionenkörpern. Nachr. Ges. Wiss. Göttingen, Math.-Phys. Kl., 1933:335-339, 1933. 
Ananyo Dan

Basque Centre for Applied Mathematics

Alameda de Mazarredo 14

48009 Bilbao, Spain

Email: adan@bcamath.org

\section{Inder Kaur}

Pontifícia Universidade Católica do Rio de Janeiro (PUC-Rio)

R. Marquês de São Vicente, 225

Gávea, Rio de Janeiro - RJ, 22451-900, Brasil

Email: inder@mat.puc-rio.br 